\title{
Percepção da qualidade de serviços nas bibliotecas da Universidade Federal de Ouro Preto na perspectiva do usuário
}

\section{Luciana Matias Felício Soares}

\author{
Graduado em Ciências da Informação e Mestre \\ em Administração pela Faculdade Novos \\ Horizontes
}

Caissa Veloso e Sousa

Economista, Mestre e ecoutora em
Administração. Professora e Pesquisadora do
Mestrado Acadêmico em Administração da
Faculdade Novos Horizontes

http://dx.doi.org/10.1590/1981-5344/2050

Este estudo tem como objetivo identificar e analisar a percepção dos usuários do Sistema de Bibliotecas e Informação na Universidade Federal de Ouro Preto no Estado de Minas Gerais, acerca da qualidade dos serviços prestados por essas bibliotecas. Para atender a esse objetivo adaptou-se a escala Servqual desenvolvida por Parasuraman, Zeithaml e Berry (2006) e o modelo Libqual desenvolvido por Cook, Thompson e Heath (2004), para a elaboração do questionário. A presente pesquisa é de abordagem quantitativa e quanto aos fins, se enquadra como descritiva. Quanto aos meios, a pesquisa se qualifica como um estudo de caso. Os dados dos questionários foram computados estatisticamente por meio de técnicas de análise univariadas, como a estatística descritiva e o teste não paramétrico de Friedmann. Nos resultados do estudo pode-se destacar que, em relação a expectativa dos serviços, os usuários esperam que as bibliotecas cumpram o que é prometido em relação aos atributos dos serviços, além disso, que os funcionários estejam dispostos a auxiliar os usuários e que os funcionários transmitam conhecimento e cortesia. Na percepção dos usuários, os serviços oferecidos pelas bibliotecas demonstram ser confiáveis e os funcionários auxiliam os usuários nas dúvidas em relação a utilização dos serviços das bibliotecas. Ainda em relação a percepção dos serviços, os usuários apresentaram 
insatisfação em relação aos equipamentos e infraestrutura das bibliotecas. Comparando os valores das diferenças entre expectativa e percepção, observou-se que em nenhuma dimensão as percepções superam as expectativas, o que enseja que devem ser realizadas melhorias em vários aspectos na prestação dos serviços das bibliotecas do Sisbin para que os serviços sejam considerados de qualidade pelos usuários.

Palavras-chave: Qualidade de serviços; Servqual; Libqual; Bibliotecas Universitárias.

\section{Perceptions of quality of services of libraries in the UFOP user perspective}

This study aims to identify and analyze the perception of the users of the System of Libraries and Information of the Federal University of Ouro Preto in the State of Minas Gerais, about the quality of the services provided by these libraries. To meet this objective it has adapted the Servqual scale developed by suggested by Parasuraman, Zeithaml and Berry (2006) and the Libqual model developed by Cook, Thompson and Heath (2004), for the preparation of the questionnaire. The present research is quantitative approach and about the purposes, it fits as descriptive. As to the means, research qualifies as a case study. The data from the questionnaires were computed statistically by meannings of univariate analysis techniques, such as the descriptive statistics and the nonparametric test of Friedmann. The results of the research may be pointed out that in relation to expectations of service users expect that libraries fulfill what is promised in relation to the attributes of the services, in addition, that employees are willing to assist users and employees to transmit cortesia. Na knowledge and perception of the users the services offered by libraries prove to be reliable and the staff assist users with questions regarding the use of library services. Also in relation to the perception of the services, users showed dissatisfaction with the equipment and infrastructure of libraries. Comparing the values of the differences between expectation and perception, it was observed that in any dimension perceptions exceed expectations, which entails that must be made improvements in several aspects in the provision of 
services of Sisbin libraries so that services are considered quality by users.

Keywords: Quality of services; Servqual; Libqual; University Libraries.

Recebido em 24.03.2014 Aceito em 12.05.2015

\section{Introdução}

Assim como nas empresas privadas, a administração pública deve acompanhar as demandas da sociedade por novos produtos e serviços com maior qualidade e eficiência. As reformas administrativas ocorridas no Brasil, principalmente a partir de 1994, na primeira gestão do governo de Fernando Henrique Cardoso, vêm ao encontro desse objetivo, tendo como principais metas melhorar a qualidade dos serviços na administração pública e atender às demandas do cidadão. "Essas novas práticas são conhecidas como New Public Management (NPM) ou Administração Pública Gerencial (AP Gerencial)" (DI GIACOMO, 2005, p. 156).

Essa preocupação por parte do governo em relação à qualidade está presente na educação, por meio de ações como o Plano de Desenvolvimento da Educação (PDE), criado em 2007 pelo Ministério da Educação, no governo de Luiz Inácio Lula da Silva, então presidente da república, e do ministro da Educação Fernando Haddad. O PDE abarca quatro eixos estruturais, que são: a educação básica, educação superior, educação profissional e alfabetização (BRASIL, [200-?]).

Compondo esse contexto, a biblioteca é um dos aportes importantes da educação, pois ela faz parte de uma das categorias de avaliação do Sistema Nacional de Avaliação da Educação Superior (Sinaes). Especificamente na categoria instalações físicas, as bibliotecas se destacam, pois, dentre os dez indicadores que compõem essa dimensão, três se referem à biblioteca, o que demonstra sua importância na avaliação de instituições de ensino superior (BRASIL, 2009).

A partir dos parâmetros das avaliações dos cursos, os gestores responsáveis pelos serviços prestados pelas bibliotecas passaram a se preocupar, de forma sistemática, com a qualidade desses serviços, visando se adequar aos quesitos exigidos na avaliação. A avaliação da qualidade de serviços é uma ferramenta estratégica para a gestão de Unidades de Informação como as Bibliotecas, sendo uma etapa importante para melhoria dos serviços prestados por esses órgãos, pois o planejamento permite elaborar políticas e diretrizes para alcançar o objetivo almejado (LANCASTER, 1996). Uma das formas de avaliar a qualidade dos serviços oferecidos consiste em verificar se os clientes das bibliotecas percebem que esses serviços atendem a suas demandas e necessidades de informação, o chamado foco no cliente, que procura 
aproximar o fornecedor de produto/serviço e seus clientes (VERGUEIRO, 2002).

Hoffman et al. (2009) destacam que no planejamento de serviços, geralmente, os gerentes levam em consideração apenas a visão do gestor sobre como deve ser um serviço de qualidade, o que gera a desconexão entre os atributos que o gerente julga necessários nos serviços e os serviços que os clientes julgam que atenderão as suas expectativas.

Neste contexto tem-se o seguinte questionamento, que norteou o trabalho: Qual a percepção dos usuários acerca da qualidade dos serviços prestados em bibliotecas.

Como objetivo o estudo pretendeu identificar e analisar a percepção dos usuários do Sistema de Bibliotecas e Informação na Universidade Federal de Ouro Preto no Estado de Minas Gerais, acerca da qualidade dos serviços prestados por essas bibliotecas.

\section{Qualidade de serviços e estudos de usuários}

Nesta seção apresentam-se as temáticas que sustentam os argumentos de pesquisa. São tratados os temas: qualidade dos serviços; escalas Servqual e Libqual, que mensuram a qualidade dos serviços; além disso, discute-se sobre estudos de usuários.

\subsection{Qualidade de serviços}

O termo qualidade pode ser definido de várias formas. Para Garvin (1992), a qualidade pode ser conceituada em cinco aspectos: primeiro, transcendente, considerada "excelência inata", busca pelo alto padrão amplamente reconhecível; segundo ligada a um produto, a qualidade pode ser medida e precisa; terceiro, o foco no usuário, busca-se a qualidade atendendo as demanda e necessidades do consumidor, enfoque subjetivo, quarto, a qualidade baseada na produção, fundamentada na oferta e nos pressupostos da engenharia e da produção, sendo assim, o produto ou serviço deve seguir padrões específicos com o objetivo principal de reduzir os custos e quinto, a qualidade baseada no valor que envolve a excelência e o preço.

Em relação aos serviços, Lovelock e Wirtz (2003) os definem como "um ato ou desempenho que cria benefícios para clientes por meio de uma mudança desejada no - ou em nome do - destinatário do serviço" (LOVELOCK; WIRTZ, 2003, p.5). Ainda segundo os autores, as diferenças entre bens e serviços não são muito bem definidas, sendo que as primeiras pesquisas realizadas definiam diferenças genéricas dos serviços em relação aos bens como intangibilidade, perecibilidade, simultaneidade e heterogeneidade. Nessa linha de pensamento, autores como Hoffman et al. (2009) e Zeithaml e Bitner (2003) identificaram essas características dos serviços que dificultam a avaliação da qualidade de serviços, estas características são detalhadas como: 
a)Intangibilidade: falta de elementos tangíveis, pois não podem ser tocados, estocados, patenteados e dificuldade em seu deslocamento para atender demandas súbitas;

b)Inseparabilidade: a produção e o consumo são simultâneos e o cliente participa de várias formas, ele pode participar de todas as fases do processo ou em partes especificas como o início ou o fim do processo. Além disso, a presença de outros clientes afetam os serviços e os funcionários afetam o resultado dos serviços;

c)Heterogeneidade: dificuldade em padronizar e controlar os serviços, pois este varia de cada funcionário que presta o serviço;

d)Perecibilidade: dificuldade em armazenar os serviços para serem utilizados em outro momento impossibilitando o planejamento em longo prazo dos serviços.

Outro aspecto importante relativo à qualidade de serviço repousa sobre as dificuldades dos clientes para avaliar serviços muito especializados como, por exemplo, serviços legais, serviços de arquitetura e serviços educacionais, e acabam utilizando como indicadores de julgamento da qualidade atributos ligados às características interpessoais dos prestadores de serviço (ZEITHAML; BITNER, 2003).

Sobre a qualidade de serviços , estudos foram realizados na área de ciência da informação, algumas pesquisas abordam indicadores de qualidade como os trabalhos de Rebello (2004) que apresenta um relato de experiência sobre a satisfação do usuário em relação aos serviços prestados pela Biblioteca e Documentação Científica do Hospital Universitário da Universidade de São Paulo (SBDC-HU/USP). Ainda relacionado aos indicadores, Rozados (2005) desenvolveu trabalho teórico que aborda a mensuração da qualidade de unidades de informação com foco no usuário, por meio de indicadores de desempenho recomendados nas normas ISO 11620 e ISO 20983 (INTERNATIONAL ORGANIZATION FOR STANDARDIZATION, 2003a e 2003b).

Além disso, vários trabalhos foram publicados com o tema qualidade de serviços tendo como ferramenta principal a escala Servqual como as pesquisas de Sampaio et al. (2004), Igami, Sampaio e Vergueiro (2005), Freitas, Bolsanello e Viana (2008), e Brito e Vergueiro (2011).

\subsection{Escala servqual}

Para identificar aspectos da qualidade dos serviços, Parasuraman, Zeithaml e Berry (2006) desenvolveram um modelo conceitual de qualidade de serviços. Este se divide em cinco lacunas: lacuna 1, lacuna da compreensão do cliente, lacuna 2, lacuna do projeto e dos padrões de serviço, lacuna 3, lacuna do desempenho do serviço, lacuna 4, lacuna da comunicação e lacuna 5, a lacuna do cliente (ZEITHAML; BITNER; GREMLER, 2011). Esta pesquisa tem como foco estudar a lacuna 5 do cliente, que é considerada a base do modelo de lacunas, esta consiste na diferença entre a expectativa versus a percepção. A expectativa advém do ponto de referencia do cliente, ou seja, suas experiências anteriores em 
relação ao tipo de serviço ofertado e a percepção dos serviços são avaliações sobre os serviços recebidos, conforme mostra Figura 1.

Figura 1 - A lacuna do cliente

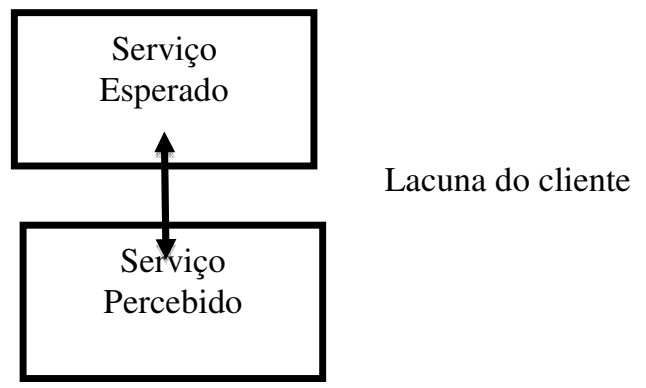

Fonte: ZEITHAML, BITNER E GREMLER (2011, p. 69).

O ideal na avaliação da qualidade de serviços compreende em igualar os quocientes da expectativa dos clientes com a percepção dos mesmos, ou seja, que a diferença entre eles seja nula ou com pouca variação. Segundo Zeithaml, Bitner e Gremler (2011), as expectativas dos clientes são controladas pelo mercado, por meio de propaganda e preço, além disso, envolvem questões como a necessidade dos clientes, as ofertas e a comunicação boca a boca.

Com base no modelo de lacunas, Parasuraman, Zeithaml e Berry (2006) desenvolveram a escala Servqual que é um instrumento com a finalidade de medir a qualidade de serviços composto por 44 questões fechadas, sendo que destas, 22 questões referem-se à expectativa do cliente e 22 questões avaliam a percepção, ou seja, a qualidade do serviço recebido. Estas questões são distribuídas em cinco dimensões: confiabilidade, responsividade, segurança, empatia e tangíveis (ZEITHAML; BITNER; GREMLER, 2011).

A dimensão da confiabilidade consiste na capacidade de cumprir o que é prometido em relação aos atributos do serviço, padronização de como o serviço é oferecido. Esta dimensão é importante, pois, caso não seja cumprido o serviço almejado, o cliente perde a confiança na empresa e muda de prestadora (HOFFMAN et al., 2009; ZEITHAML; BITNER; GREMLER, 2011).

A dimensão da responsividade envolve a disposição da empresa em auxiliar os clientes, oferecendo serviço de acordo com a necessidade do cliente e envolve o tempo de espera pelo serviço (HOFFMAN et al., 2009; ZEITHAML; BITNER; GREMLER, 2011).

A dimensão da segurança está relacionada às características que os funcionários transmitem, como conhecimento e cortesia, juntamente com a competência da empresa e a segurança das operações, que geram a confiança e certeza no cliente (HOFFMAN et al., 2009; ZEITHAML; BITNER; GREMLER, 2011). 
A dimensão da empatia envolve o cuidado, a atenção personalizada e a customização dos serviços, que geram no cliente a sensação de que os mesmos são especiais para empresa, possibilitando relacionamentos mais próximos aos clientes e maior satisfação (HOFFMAN et al., 2009; ZEITHAML; BITNER; GREMLER, 2011)

A dimensão da tangibilidade consiste em objetos tangíveis, representações físicas que auxiliam na prestação do serviço, como equipamentos, instalações, pessoal e material de comunicação (HOFFMAN et al., 2009; ZEITHAML; BITNER; GREMLER, 2011).

\subsection{Escala Libqual}

A escala Libqual foi desenvolvida pela Association of Research Libraries (ARL) em parceria com a Texas A\&M University (Tamu), com a colaboração dos pesquisadores Cook, Thompson e Heath. A pesquisa resultou no desenvolvimento da escala e recebeu apoio financeiro da U.S. Department of Education's Fund for the Improvement of Postsecondary Education (Fipse) (BLIXRUD, 2003). A Libqual é um instrumento adaptado para biblioteca com base no modelo Servqual, cuja finalidade era tornar a ferramenta mais adequada para o ambiente da biblioteca, pois a biblioteca não tem a finalidade comercial e lucrativa (BRITO; VERGUEIRO, 2012).

Morales et al. (2011), afirmam que o instrumento original da Libqual contemplava 41 perguntas subdivididas em cinco dimensões: valor afetivo do serviço "affect of service", biblioteca como lugar "library as place", confiabilidade "reliability", disponibilidade de acervos físicos "provision of physical collections", e acesso à informação "information access". Com o decorrer de várias experiências, o instrumento foi refinado para três dimensões em 2004.

Atualmente a Libqual é um instrumento composto por 22 questões, com módulos opcionais de comentários livres e questões demográficas. Além disso, podem ser utilizadas cinco questões ligadas à competência informacional, sendo assim, compreende um total de 27 questões. Tal instrumento ainda possibilita o acesso à versão Libqual Lite, que é uma forma simplificada contendo oito questões. Para ter acesso à ferramenta completa de pesquisa no Libqual, o usuário ou a biblioteca deve efetuar o pagamento de uma taxa (BRITO; VERGUEIRO, 2012). Ainda segundo Brito e Vergueiro (2012), a versão atual da Libqual possui três dimensões (valor afetivo do serviço, controle da informação e biblioteca como lugar).

$\mathrm{Na}$ dimensão valor afetivo do serviço, observam-se os aspectos humanos da qualidade dos serviços; a dimensão controle da informação envolve aspectos como suporte da informação, facilidade de acesso à informação de forma autônoma. Na dimensão biblioteca, como lugar físico, percebem-se aspectos ligados às características físicas e tangíveis das bibliotecas (BRITO; VERGUEIRO, 2012).

Dentre os vários estudos realizados com o instrumento Libqual, destaca-se a pesquisa de Morales et al. (2011), pois tinha como objetivo avaliar a versão em espanhol da Libqual nos aspectos como sua estrutura, 
confiabilidade e validade. A metodologia do estudo contemplou a aplicação de 374 questionários em uma universidade mexicana, eles concluíram que o instrumento Libqual é um instrumento consistente.

Ressalta-se que nesta pesquisa a escala Libqual não foi utilizada na integra, pois não está disponível para utilização gratuita e como a pesquisa foi realizada em uma instituição pública, privilegiou-se a utilização de ferramentas que podem ser replicadas em pesquisas futuras.

\subsection{Estudos de usuários}

Estudar os serviços das bibliotecas universitárias consiste em compreender o uso e necessidade de informação. Para tal, torna-se importante descrever os estudos de usuários em bibliotecas que, para Figueiredo (1994),

são investigações que se fazem para saber o que os indivíduos precisam em matéria de informação, ou então, para saber se as necessidades de informação por parte dos usuários de uma biblioteca ou de um centro de informação estão sendo satisfeitas de maneira adequada (FIGUEIREDO, 1994, p. 7).

Ainda para a autora, esses estudos procuram entender como, por que e para quais fins os usuários buscam e utilizam a informação. De posse destes elementos, as bibliotecas podem verificar as demandas dos usuários e se as mesmas são atendidas de forma adequada.

As principais correntes teóricas de estudos de usos e usuários da informação podem ser dividas em duas abordagens, a tradicional e a alternativa. A abordagem tradicional, segundo Choo e Rocha (2003), teve início em pesquisas realizadas no ano de 1948, na Conferência sobre Informação Científica, da Royal Society, na qual foram apresentados dois estudos: o primeiro abordava o comportamento de busca da informação de cientistas britânicos do órgão do governo, e o segundo, o uso da biblioteca do Museu de Ciência de Londres.

A literatura da área aponta que os pesquisadores dessa corrente concentravam seus estudos no entendimento dos sistemas, das comunidades e eram orientados para a tarefa. Além disso, tais estudos são apontados como pesquisas de aplicação utilitária, independente do usuário e objetivas, sendo válidas para avaliar produtos e serviços (CUNHA, 1982; PINHEIRO, 1982, TAYLOR, 1986).

Figueiredo (1994) argumenta que foram feitas críticas às limitações dos estudos de usuários realizados nessa linha de pensamento. Uma delas consiste na adoção de novas metodologias de estudo, visto que os esforços desses estudos se voltam para pesquisas empíricas de caráter funcionalista, o que também foi observado por Lima (1992) e Araújo (2010). Além disso, Figueiredo (1994) acrescenta que os estudiosos sugeriram a criação de modelos teóricos e conceituais sobre o uso da informação, sendo outro problema levantado a dificuldade de estabelecer 
uma relação efetiva entre a obtenção de um documento e o uso efetivo da informação.

A partir dessas críticas, surgiu a abordagem alternativa nos estudos de usuários cujo foco principal é o usuário. Segundo Choo e Rocha (2003), nessa abordagem, a informação é uma construção subjetiva que passa por processos cognitivos da mente do usuário, permitindo criar significado para a informação, tornando-a útil.

A abordagem alternativa abarca estudos centrados nos usuários, que levam em consideração a percepção de produtos e serviços das bibliotecas. Esses estudos, segundo Dervin e Nilan (1986), citados por Ferreira (1997), se caracterizam por: considerar o indivíduo como ser construtivo e ativo, inserido em um contexto, sem deixar de levar em consideração a individualidade e os processos cognitivos envolvidos nos processos, a visão holística e a orientação qualitativa dos estudos.

Além disso, segundo Ferreira (1997), essa abordagem se desenvolve em várias vertentes:
a)Abordagem de "Valor Agregado" de Robert Taylor (1986);
b)Abordagem do "Estado de Conhecimento Anômalo" de Belkin e Oddy e Brooks (1982);
c)Abordagem do "Processo Construtivista" de Carol Kuhlthau (1993);
d)Abordagem "Sense-Making" de Brenda Dervin (1977,1983, 1993,1994).

Dentre as abordagens acima, a abordagem Sense-making destacase por mapear a necessidade de informação sob o ponto de vista do usuário, visto que envolve vários elementos em suas análises a partir da percepção e compreensão do mundo interior e exterior ao usuário.

O modelo de Dervin (1992) tem como suporte a teoria de vários estudiosos de diversos campos que influenciaram sua base teóricometodológica. Dentre elas a principal é de Carter (1967) citado por Ferreira (1997), que desenvolveu estudos na área de comunicação. O autor afirma que o homem se depara com obstáculos, descontinuidades, que dificultam a compreensão de algo, a chamada lacuna (gap) e esta falha leva o indivíduo a buscar informação para voltar a seu caminho ou estado normal (FERREIRA, 1997).

Ainda sobre a necessidade e uso da informação, surgiram recentemente novos modelos como o modelo de Wilson (1981) que foi reformulado em 1996 e a percepção cognitivista de Choo e Rocha (2003) que propõe um modelo multifacetado de uso da informação.

O modelo de Wilson (1981) tem como base os fatores as necessidades fisiológicas, cognitivas e afetivas dos usuários, porém em estudos realizados pelo autor em 1996 apontaram fragilidades nos construtos conceituais do comportamento de informação, pois estes 
ignoraram modelos mais robustos sobre comportamento humano de áreas correlatas. Sendo assim, Wilson (1999) realizou uma revisão bibliográfica sobre as pesquisas em comportamento da informação o que possibilitou alteração em seu modelo tornando-o mais abrangente ao abarcar diversas áreas do conhecimento (tomada de decisões, psicologia, inovação, comunicação em saúde e pesquisa do consumidor), indo além do comportamento de busca de informação e necessidades de informação.

O modelo da percepção cognitivista de Choo e Rocha (2003) propõe uma perspectiva multifacetada de uso da informação que se divide conceitualmente em três estágios: necessidade de informação, busca de informação e uso da informação.

A necessidade de informação passa pelo primeiro estágio visceral, ocasionando sensação de incerteza e intranquilidade; no segundo estágio transforma-se em questões ou temas conscientes que auxiliam na busca de informações e no terceiro acaba se formalizando.

A busca de informação tenta compreender o comportamento dos indivíduos no processo de busca de informação, esse processo sofre interferência de fatores emocionais, situacionais e cognitivos (CHOO; ROCHA, 2003).

E o uso da informação consiste na maneira de utilização das respostas que pode servir para responder a uma questão, solucionar problema ou tomar uma decisão alterando o conhecimento do indivíduo. Depende de processos como a expectativa do grupo que o indivíduo pertence e pode promover sensações de satisfação, confiança, decepção ou frustração (CHOO; ROCHA, 2003).

\section{Procedimentos metodológicos}

A presente pesquisa é de abordagem quantitativa (COLLIS, HUSSEY, 2005), quanto aos fins, se enquadra como descritiva (VERGARA, 2003; TRIVIÑOS, 1987). Quanto aos meios, a pesquisa se qualifica como um estudo de caso que foi realizado na Universidade Federal de Ouro Preto (UFOP), mais especificamente no Sistema de Bibliotecas e Informação (SISBIN). Optou-se pelo estudo de caso, pois este possibilita compreender de forma contextualizada a percepção dos usuários sobre a qualidade dos serviços nas bibliotecas setoriais do Sisbin da UFOP. (GIL, 2002; TRIVIÑOS,1987; VERGARA, 2003).

A população-alvo desta pesquisa é composta pelos usuários do Sisbin, que são os alunos, professores e funcionários, ou seja, a comunidade acadêmica que utiliza os serviços oferecidos pelas bibliotecas. A amostra foi não-probabilística por conveniência. Segundo Hair Jr. et al. (2005, p. 246), "nas amostras não-probabilísticas, a seleção de elementos para a amostra não é necessariamente feita com o objetivo de ser estatisticamente representativo da população".

Do universo de 19.848 usuários foram coletados 325 questionários, entre os dias 7 e 17 de outubro de 2013. O questionário foi composto por questões adaptadas da escala Servqual e da dimensão controle da 
informação da escala Libqual, sendo que cada questionário continha 53 questões no total.

Os dados foram analisados por meio de técnicas univariadas, como a estatística descritiva e como não foi encontrada normalidade dos dados coletados foram utilizados os testes não-paramétricos de Friedmann, Wilcoxon, Mann-Whitney, Kruskall-Wallis (TRIOLA, 1998; DOWNING; CLARK, 2010; MALHOTRA, 2006). Além disso, os dados da pesquisa foram tratados por meio da utilização de planilha eletrônica Excel 2010 e do programa estatístico $R$ Core Team 2013 (R), de forma a obter dados estatísticos descritivos que caracterizem a amostra. Ressalta-se ainda que esta pesquisa foi submetida ao Comitê de Ética em Pesquisa (CEP UFOP), para aprovação, formalizando-se pelo parecer 406.820 e CAAE: 13004113.5 .0000 .5150$.

\section{Apresentação e análise dos resultados}

A seguir apresenta-se a distribuição dos respondentes por: frequência de utilização das bibliotecas, sexo, faixa etária, renda média familiar, estado civil, formação acadêmica e ocupação.

Observou-se que $16,30 \%$ (53) dos usuários declarou frequentar a biblioteca diariamente, 24,61\% (80) dos usuários frequentam a biblioteca uma vez por semana, $31,07 \%$ (101) dos usuários frequentam a biblioteca de duas a três vezes por semana e 28,00\% (91) dos usuários frequentam a biblioteca entre duas a três vezes por mês.

Em relação ao sexo, percebe-se homogeneidade entre os respondentes do sexo masculino e feminino, sendo $163(50,2 \%)$ do sexo masculino e $162(49,8 \%)$ do sexo feminino.

A maioria dos respondentes, 72,0\% (234 pessoas) tem entre $19 \mathrm{e}$ 25 anos de idade, sendo que 11,4\% (37) dos usuários possuem entre 26 e 30 anos de idade. Observa-se, ainda, que 5,6\% (18) dos usuários possuem mais de 40 anos, 5,2\% (17) possuem até 18 anos, 3,0\% (10) possuem entre 36 e 40 anos de idade e 2,8\% (9) possuem entre 31 e 35 anos de idade.

A grande maioria dos pesquisados, 68,9\% (224), provém de famílias com renda inferior a $R \$ 4.746,00$, sendo mais frequente a presença de usuários cuja renda familiar encontra-se entre $R \$ 2.712,00$ e $R \$$ 4.746,00.

Em função de o público alvo das bibliotecas ser constituído por estudantes de graduação, 89\% (289) dos respondentes se declararam solteiros. Observa-se, ainda, que 10,4\% (34) dos respondentes se declararam casados ou em união estável, $0,3 \%$ (1) respondente se declarou divorciado ou separado e 0,3\% (1) viúvo.

Em relação formação acadêmica, pode-se observar que a maioria dos respondentes 92,6\% (301), são estudantes de graduação, sendo que, entre os demais, destaca-se a participação de usuários com especialização completa $2,5 \%(8)$. 
Sobre o cargo ou função exercida, a maioria dos respondentes, 90,8\% (296 pessoas), se declararam estudantes de graduação e 5,0\% (16) dos respondentes são funcionários da biblioteca que se declararam não bibliotecários. Observa-se, ainda, que 2,2\% (7) se declararam como do corpo docente, $1,6 \%$ (5) são funcionários técnico-administrativo e $0,4 \%$ (1) se declarou na categoria "demais funcionários".

\subsection{Análise univariada da escala servqual}

Nesta seção são analisados os dados referentes à escala Servqual e Libqual que corresponde à segunda etapa do questionário, contendo 23 questões referentes às expectativas e 23 questões referentes às percepções dos serviços prestados pelas bibliotecas do Sisbin. Para efeito de apresentação, procurou-se agrupar essas variáveis em cinco dimensões, conforme mostra o Quadro 1.

Quadro 1 - Distribuição da análise das questões do questionário

\begin{tabular}{cll}
\hline Escala & \multicolumn{1}{c}{ Dimensão } & \multicolumn{1}{c}{ Questões } \\
\hline Servqual & Confiabilidade & $1,2,6$ \\
& Responsividade & $3,4,5,7$ \\
& Segurança & $8,9,10$ \\
& Empatia & $13,14,15$ \\
& Tangíveis & $16,17,18,22$ \\
& & \\
Libqual & Controle da informação & $11,12,19,20,21,23$ \\
\hline
\end{tabular}

Fonte: Dados da pesquisa.

No instrumento questionário, a construção da escala de múltiplos itens adotou-se o padrão Likert de 7 pontos, que permite a graduação das repostas com variação entre 'discordo totalmente' e 'concordo totalmente'. Sendo assim, nesta pesquisa, considerou-se como escore médio ou mediano o valor de 4,0 a 4,99, sendo que as respostas que apresentarem escores acima de 4,99 indicam uma situação de concordância, abaixo de 4,0 uma situação de discordância e, igual a este valor, uma situação intermediária entre a concordância e a discordância.

O questionário foi submetido a um pré teste, com $10 \%$ da amostra, conforme sugere Hair Jr. et al. (2005). No pré teste constatou-se a superioridade da escala de 7 pontos em detrimento da escala de 5 pontos. Os demais dados permaneceram inalterados.

A seguir são apresentados os dados da pesquisa por dimensões; foram utilizadas para essa apresentação, medidas de posição como a média e mediana, desvio padrão e o intervalo interquartil $\left(P_{25}\right.$ e $\left.P_{75}\right)$. 
Tabela 1 - Caracterização dos usuários sobre a qualidade dos serviços das bibliotecas segundo as dimensões de expectativa

\begin{tabular}{lcc|c|c|c}
\hline \multicolumn{1}{c|}{ Dimensão } & Média & $\begin{array}{c}\text { Desvio } \\
\text { Padrão }\end{array}$ & $\mathbf{P}_{\mathbf{2 5}}$ & Mediana & $\mathbf{P}_{\mathbf{7 5}}$ \\
\hline Confiabilidade & 5,34 & 1,17 & 4,66 & 5,66 & 6,00 \\
Responsividade & 5,60 & 0,94 & 5,00 & 5,75 & 6,25 \\
Segurança & 5,41 & 1,04 & 4,66 & 5,66 & 6,00 \\
Empatia & 4,88 & 1,27 & 4,00 & 5,00 & 5,66 \\
Tangíveis & 4,32 & 1,43 & 3,25 & 4,25 & 5,25 \\
Controle da informação & 4,45 & 1,30 & 3,50 & 4,33 & 5,50 \\
\hline
\end{tabular}

Fonte: Dados da pesquisa.

A Tabela 1 apresenta as estatísticas descritivas da expectativa dos respondentes em relação aos serviços, agrupadas pelas dimensões em estudo. Pode-se observar que a média das dimensões está variando entre 4,32 e 5,60, gerando uma amplitude de 1,28 entre as mesmas. Observase que as dimensões empatia, tangíveis e controle da informação apresentaram situação intermediária entre discordância e concordância, porém as dimensões confiabilidade, responsividade e segurança apresentaram situação de concordância.

Destaca-se a dimensão responsividade com maior escore $(5,60)$, ou seja, os usuários esperam que a biblioteca tenha disposição em auxiliar os usuários de acordo com suas solicitações, promovendo rapidez no atendimento, informando o horário de atendimento e mostrando disponibilidade par atender as suas solicitações (HOFFMAN et al., 2009; ZEITHAML; BITNER; GREMLER, 2011).

Tabela 2 - Teste de Friedman para comparação entre as dimensões Expectativa

\begin{tabular}{l|c|c}
\hline \multicolumn{1}{c|}{ Dimensões } & Média & p-valor \\
\hline Confiabilidade & 5,34 & \\
Responsividade & 5,60 & \\
Segurança & 5,41 & $0,000^{* *}$ \\
Empatia & 4,88 & \\
Tangíveis & 4,32 & \\
Controle da informação & 4,45 &
\end{tabular}

**Nível de Significância de 0,01

Fonte: Dados da pesquisa.

A Tabela 2 apresenta o teste de Friedmann aplicado nas dimensões da expectativa dos usuários, para verificar se existem diferenças nas dimensões avaliadas pelos usuários, ou se os mesmos avaliam todas as dimensões de forma semelhante. De acordo com o teste de Friedman, com $1 \%$ de significância, têm-se razões estatísticas para concluir que a avaliação dos usuários é distinta, se verificarmos todas as dimensões da 
expectativa conjuntamente, pois o teste apresentou um valor-p de $0,000 * *$.

Tabela 3 - Caracterização dos usuários sobre a qualidade dos serviços das bibliotecas segundo as dimensões de percepção

\begin{tabular}{lcc|c|c|c}
\hline \multicolumn{1}{c|}{ Dimensão } & Média & $\begin{array}{c}\text { Desvio } \\
\text { Padrão }\end{array}$ & $\mathbf{P}_{\mathbf{2 5}}$ & Mediana & $\mathbf{P}_{\mathbf{7 5}}$ \\
\hline Confiabilidade & 5,24 & 1,12 & 4,66 & 5,33 & 6 \\
Responsividade & 5,35 & 0,99 & 4,75 & 5,50 & 6 \\
Segurança & 4,99 & 1,22 & 4,00 & 5,33 & 6 \\
Empatia & 4,76 & 1,33 & 4,00 & 5,00 & 6 \\
Tangíveis & 3,90 & 1,38 & 2,75 & 4,00 & 5 \\
Controle da informação & 4,10 & 1,17 & 3,33 & 4,00 & 5 \\
\hline
\end{tabular}

Fonte: Dados da pesquisa.

A Tabela 3 apresenta as estatísticas descritivas da percepção dos respondentes em relação aos serviços, classificadas pelas dimensões em análise. Pode-se observar que a média das dimensões oscila entre 3,90 e 5,35, com amplitude de 1,44. Percebe-se que os escores médios para percepção, aparentemente, são menores, se comparados com os escores médios para expectativa.

Observa-se, ainda, que as dimensões segurança, empatia e controle da informação apresentaram situação intermediária entre discordância e concordância; as dimensões confiabilidade, responsividade apresentaram situação de concordância, porém a dimensão tangíveis apresentou situação de discordância, com menor escore 3,90.

Tabela 4 - Teste de Friedman para comparação entre as dimensões Percepção

\begin{tabular}{lc|c}
\hline \multicolumn{1}{c|}{ Dimensões } & Média & p-valor \\
\hline \hline Confiabilidade & 5,24 & \\
Responsividade & 5,35 & \\
Segurança & 4,99 & $0,000^{\star *}$ \\
Empatia & 4,76 & \\
Tangíveis & 3,90 & \\
Controle da informação & 4,10 & \\
\hline
\end{tabular}

**Nível de Significância de 0,01

Fonte: Dados da pesquisa.

A Tabela 4 apresenta o teste de Friedmann para verificar se as dimensões da percepção são igualmente avaliadas pelos usuários ou se há diferenças estatísticas entre as mesmas. De acordo com o teste de Friedman, com 1\% de significância, conclui-se que a avaliação dos usuários é distinta, se verificarmos todas as dimensões da percepção conjuntamente, pois o teste apresentou um valor-p de 0,000**. 


\subsection{Diferença entre expectativa e percepção}

Para mensurar a distância existente entre expectativa e percepção nas dimensões em estudo, foram calculadas as diferença entre as médias dos escores referentes a cada questão presente no questionário. As diferenças entre as médias estão apresentadas na Tabela 5.

Tabela 5 - Diferença entre expectativa e percepção

\begin{tabular}{|c|c|c|c|c|c|c|c|c|c|c|c|c|c|c|c|c|c|c|c|c|c|c|c|}
\hline \multicolumn{24}{|c|}{ Dimensões da Qualidade } \\
\hline & \multicolumn{3}{|c|}{ Confiabilidade } & \multicolumn{4}{|c|}{ Responsividade } & \multicolumn{3}{|c|}{ Segurança } & \multicolumn{3}{|c|}{ Empatia } & \multicolumn{4}{|c|}{ Tangíveis } & \multicolumn{6}{|c|}{ Controle da Informação } \\
\hline & Q1 & Q2 & Q6 & Q3 & Q4 & Q5 & Q7 & Q8 & Q9 & Q10 & Q13 & Q14 & Q15 & Q16 & Q17 & Q18 & Q22 & Q11 & Q12 & Q19 & Q20 & Q21 & Q23 \\
\hline$P$ & 5,29 & 5,18 & 5,26 & 5,70 & 4,92 & 5,48 & 5,30 & 5,20 & 4,76 & 5,02 & 4,52 & 4,72 & 5,05 & 4,06 & 4,00 & 3,67 & 3,91 & 4,57 & 3,90 & 3,31 & 4,04 & 4,04 & 4,75 \\
\hline E & 5,41 & 5,28 & 5,33 & 6,00 & 5,20 & 5,72 & 5,52 & 6,03 & 4,90 & 5,30 & 4,58 & 4,73 & 5,36 & 4,53 & 4,38 & 4,18 & 4,23 & 5,05 & 4,27 & 3,69 & 4,41 & 4,38 & 4,95 \\
\hline G & 0,13 & 0,10 & 0,07 & 0,30 & 0,28 & 0,24 & 0,22 & 0,83 & 0,13 & 0,29 & 0,06 & 0,01 & 0,31 & 0,47 & 0,38 & 0,51 & 0,32 & 0,47 & 0,37 & 0,38 & 0,37 & 0,35 & 0,21 \\
\hline
\end{tabular}

Fonte: Dados da pesquisa.

Comparando as diferenças entre a expectativa e a percepção, a dimensão confiabilidade apresenta os valores mais baixos. Essa dimensão corresponde às questões 1,2 e 6, que abordam respectivamente a informação sobre o prazo dos serviços e suas alterações, com diferença de 0,13 ; a biblioteca demonstrar interesse em resolver os problemas no atendimento ao usuário com diferença de 0,10 e os funcionários demonstrarem interesse em resolver os problemas dos usuários com diferença de 0,07. Sendo assim, entende-se que, na dimensão da confiabilidade, as bibliotecas do Sisbin estão próximas a atender as expectativas dos usuários, porém nenhuma questão atende as necessidades dos usuários satisfatoriamente.

$\mathrm{Na}$ dimensão responsividade, a questão 7 , que se refere à disponibilidade dos funcionários para atender as solicitações dos usuários, apresentou menor diferença entre expectativa e percepção, com 0,22, portanto pode-se inferir que este quesito está mais próximo de atender a necessidade dos usuários. Porém, a questão 3 , que versa sobre a execução do serviço de empréstimo com rapidez, apresentou maior diferença, com 0,30, sinalizando que este quesito é crítico para essa dimensão e deve ser melhorado. A questão 5 (informações acerca do horário de funcionamento dos serviços) e 4 (empréstimo de materiais de outras bibliotecas da UFOP é rápido) apresentaram, respectivamente, diferença de 0,24 e 0,28. Contudo, observa-se que nenhuma questão desta dimensão atende as expectativas dos usuários satisfatoriamente, evidenciando a necessidade de melhorias.

A dimensão segurança apresenta a maior diferença entre expectativa e percepção, com 0,83 na questão 8 , que procurou identificar se o comportamento dos funcionários inspira confiança no atendimento ao usuário. Porém, a questão 9, que versa sobre os funcionários serem gentis o tempo todo, apresentou menor diferença nesta dimensão com 
0,13 . Além disso, a questão 10 , que trata do conhecimento e do domínio dos funcionários sobre os serviços prestados, apresentou 0,29 de diferença. Todavia, essa dimensão também não contempla as necessidades dos usuários, evidenciando a necessidade de melhorias.

A dimensão empatia apresenta a menor diferença entre expectativa e percepção, com 0,01 na questão 14 , que versa sobre o entendimento das necessidades específicas dos usuários. Observa-se que esta questão está próxima de atender as necessidades dos usuários, porém, a questão 15, que compreende o horário de funcionamento atender as necessidades dos usuários, apresentou maior diferença, com 0,31 nesta dimensão e a questão 13, que versa sobre atenção individualizada aos usuários, apresentou diferença de 0,06.

A dimensão 'tangíveis' compreende questões com as maiores diferenças entre a expectativa e a percepção dos serviços; a questão 18, que versa sobre as bibliotecas possuírem equipamentos modernos, apresenta a maior diferença nesta dimensão com 0,51 e a questão 22, que corresponde à disponibilidade de computadores suficientes para consulta, apresenta a menor diferença com 0,32 , seguida pela questão 17 (fornecimento de material que explique os serviços oferecidos de forma clara e de fácil entendimento) e 16 (instalações confortáveis e visualmente atraentes), apresentam, respectivamente, 0,38 e 0,47 de diferença.

A última dimensão, 'controle da informação', na questão 23 , que versa sobre encontrar no lugar informado o material disponível, apresentou menor diferença entre expectativa e percepção com 0,21, portanto pode-se inferir que este quesito está mais próximo de atender a necessidade dos usuários. Porém, a questão 11 , que versa sobre o website ser funcional, apresentou maior diferença com 0,47, sinalizando que esse quesito é crítico para esta dimensão e deve ser melhorado. A questão 12 (treinamento para acesso à informação), 19 (quantidade suficiente de exemplares de livros), 20 (acervo de livros impressos atualizado) e 21 (acervo de revistas online atualizado) apresentaram, respectivamente, diferença de 0,37, 0,38,0,37 e 0,35.

Comparando todas as questões pode-se observar que a expectativa supera a percepção dos serviços em todos os quesitos. A maior diferença ocorreu na dimensão da segurança, questão 8 , que versa sobre 0 comportamento dos funcionários inspirar confiança no atendimento ao usuário, com diferença de 0,83, e a menor diferença com valor de 0,01 na questão 14 , que versa sobre os funcionários da biblioteca entenderem as necessidades específicas dos usuários.

Outras questões que apresentaram baixa diferença entre a expectativas e a percepção dos serviços foram as questões 9 da dimensão segurança, esta versa sobre a gentileza no atendimento o tempo todo, com 0,13 de diferença, e a questão 13 , com diferença de 0,06, esta versa sobre a atenção individualizada. 


\section{Considerações finais}

Neste estudo procurou-se identificar e analisar a percepção dos usuários do Sistema de Bibliotecas e Informação na Universidade Federal de Ouro Preto no Estado de Minas Gerais, acerca da qualidade dos serviços prestados por essas bibliotecas. Para tanto foram realizados estudos utilizando técnicas univariadas, como a estatística descritiva e os testes não-paramétricos de Friedmann, Wilcoxon, Mann-Whitney, Kruskall-Wallis.

Os resultados do estudo demostraram, em relação a expectativa dos serviços, que as médias das dimensões confiabilidade, responsividade e segurança foram elevadas, atingindo valores acima de 5, indicando alta expectativa nesses quesitos. Sendo assim, os usuários esperam que os serviços prestados pelas bibliotecas sejam de confiança, ou seja, que as bibliotecas cumpram o que é prometido em relação aos atributos dos serviços. Além disso, que os serviços sejam oferecidos com responsividade, ou seja, que os funcionários estejam dispostos a auxiliar os usuários e, por fim, que os serviços sejam seguros. A segurança, nesse aspecto, está relacionada as características que os funcionários transmitem, como conhecimento e cortesia, juntamente com a segurança das operações.

Porém, as dimensões empatia, tangíveis e controle da informação apresentaram valores intermediários entre 4,00 e 4,99, indicando expectativa mediana em relação aos serviços oferecidos pelas bibliotecas. Nesse sentido, os usuários apresentam expectativa intermediaria na dimensão empatia que envolve o cuidado, a atenção personalizada e a customização dos serviços. Este contexto se repete na dimensão tangíveis, ou seja, expectativa que os entrevistados têm em relação aos equipamentos, as instalações e ao material de comunicação das bibliotecas. Na dimensão controle da informação os usuários esperam receber suporte da informação e facilidade de acesso à informação de forma autônoma.

A percepção dos serviços oferecidos constitui-se em elemento chave para a avaliação da qualidade, pois envolvem vários aspectos particulares e experienciais dos indivíduos, se relacionando a um determinado serviço prestado, no caso deste trabalho os serviços prestados pelo Sisbin. Na percepção dos serviços oferecidos observou-se que as médias nas dimensões confiabilidade e responsividade foram elevadas, atingindo valores acima de 5 , indicando alta satisfação nesse quesito. Para o usuário os serviços oferecidos demonstram ser confiáveis e os funcionários auxiliam os usuários nas dúvidas em relação a utilização dos serviços das bibliotecas.

Ainda em relação à percepção dos serviços, a dimensão 'tangíveis' apresentou a menor média, com valor abaixo de 4, indicando a menor satisfação, o que leva a inferir que os equipamentos e infraestrutura das bibliotecas necessitam de investimentos para que os usuários se sintam satisfeitos no ambiente. As dimensões segurança, empatia e controle da 
informação apresentaram valores intermediários entre 4,00 e 4,99, indicando satisfação mediana.

Comparando os valores das diferenças entre expectativa e percepção, observou-se que em nenhuma dimensão as percepções superam as expectativas, o que enseja que devem ser realizadas melhorias em vários aspectos na prestação dos serviços das bibliotecas do Sisbin para que os serviços sejam considerados de qualidade pelos usuários. Porém, observou-se que na dimensão empatia na questão que versa sobre os funcionários da biblioteca entenderem as necessidades específicas dos usuários ficou próxima a atender as expectativas dos usuários. Em contrapartida a questão com a maior diferença ocorreu na dimensão da segurança, na questão que versa sobre o comportamento dos funcionários inspirar confiança no atendimento ao usuário. Para esta questão faz-se interessante realizar uma pesquisa qualitativa para verificar junto aos auxiliares de biblioteca quanto por parte dos bibliotecários esclarecer estes pontos levantados pelos usuários.

\section{Referências}

ARAUJO, C. A. A. Estudos de usuários conforme o paradigma social da Ciência da Informação: desafios teóricos e práticos de pesquisa. Informação \& Informação, Londrina, v. 15, n. 2, p. 23-39, 2010. Disponível em:

<http://www.uel.br/revistas/uel/index.php/informacao/article/view/6485/ 6995>. Acesso em: 9 maio 2015.

BLIXRUD, J. Assessing library performance: new measures, methods, and models. In: ANNUAL INTERNATIONAL ASSOCIATION OF SCIENTIFIC AND TECHNOLOGICAL UNIVERSITY LIBRARIES CONFERENCE, 24., 2003, Çankaya Ankara, Turkey. Proceedings... Çankaya Ankara: IATUL, 2003. Disponível em:

<http://docs.lib.purdue.edu/iatul/2003/papers/9>. Acesso em: 15 set. 2013.

BRASIL. Ministério da Educação. Portaria n.1, de 5 de janeiro de 2009. Diário Oficial da União, Brasília, 6 jan. 2009. p. 8. Disponível em: <http://download.Inep.gov.br/download//superior/condicoesdeensino/Extrato reconh ecimento licenciatura.pdf >. Acesso em: 26 maio 2015.

BRASIL. Ministério da Educação. Plano de desenvolvimento da educação: razões, princípios e programas. [200-?] Disponível em: <http://portal.mec.gov.br/arquivos/livro/livro.pdf>. Acesso em: 15 set. 2013.

BRITO, G. F.; VERGUEIRO, W. de C. S. Avaliação da qualidade orientada ao usuário: estudo de caso em biblioteca acadêmica utilizando o método SERVQUAL. Ciencias de laInformación, La Habana, Cuba, v. 42, n. 2, p. 55-59, maio/ago. 2011. Disponível em: <http://cinfo.idict.cu/cinfo/article/view/308>. Acesso em: 20 out. 2013. 
BRITO, G. F.; VERGUEIRO, W. de C. S. Avaliação da qualidade da

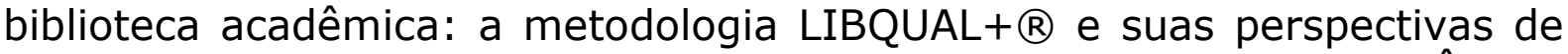
aplicação no Brasil. In: ENCONTRO NACIONAL DE PESQUISA EM CIÊNCIA DA INFORMAÇÃO,13., 2012, Rio de Janeiro. Anais... Rio de Janeiro: ENANCIB, 2012. Disponível em:

<http://www.eventosecongressos.com.br/metodo/enancib2012/arearestri ta/pdfs/19204.pdf >. Acesso em: 21 mar. 2013.

CARTER, L. F. et al. National document handling sistems for science and technology. NewYork: Willey, 1967.

CHOO, C. W.; ROCHA, E. A organização do conhecimento: como as organizações usam a informação para criar significado, construir conhecimento e tomar decisões. São Paulo: Senac, 2003.

COLLIS, J.; HUSSEY, R. Pesquisa em administração: um guia prático para alunos de graduação e pós-graduação. 2. ed. Porto Alegre: Bookman, 2005.

CUNHA, M. Metodologias para estudo dos usuários de informação científica e tecnológica. Revista de Biblioteconomia de Brasília, Brasília, v. 10, n. 2, p. 5-19, jul./dez. 1982. Disponível em: <http://bogliolo.eci.ufmg.br/downloads/CUNHA_1982.pdf>. Acesso em: 16 set. 2013.

DERVIN, B. From the mind's eye of the user: the sense-making qualitative-quatitative methodology. In: GLAZIER, J. D.; POWELL, R. R. Qualitative research in information management. Englewood, $\mathrm{CO}$ : LibrariesUnlimited, 1992. p. 61-84.

DERVIN, B.; NILAN, M. Information needs and uses. Annual Review of Information Science and Technology, v. 21, p. 3-33, 1986.

DI GIACOMO, W. A.O New Public Management no Canadá e a gestão pública contemporânea. Revista Interfaces Brasil/Canadá, Porto Alegre, RS, v. 5, n. 5, p.155-170, 2005. Disponível em:

<http://www.revistas.unilasalle.edu.br/index.php/interfaces/article/view/772/598>. Acesso em: 10 set. 2013.

DOWNING, D.; CLARK, J. Estatística aplicada. 3. ed. São Paulo: Saraiva, 2010.

FERREIRA, S. M. S. P. Estudos de necessidades de informação: dos

paradigmas tradicionais à abordagem sense-making. Porto Alegre: ABEBD, 1997.

FIGUEIREDO, N. M. Estudos de uso e usuários da informação. Brasília: IBICT, 1994.

FREITAS, A. L. P.; BOLSANELlO, F. M. C.; VIANA, N. R. N. G. Avaliação da qualidade de serviços de umabiblioteca universitária: um estudo de caso 
utilizando o modelo Servqual. Ciência da Informação, Brasília, v. 37, n. 3, p. 88-102, set./dez. 2008. Disponível em:

<http://www.scielo.br/pdf/ci/v37n3/v37n3a07.pdf>. Acesso em: 5 set. 2013.

GARVIN, D. A. Gerenciando a qualidade: a visão estratégica e competitiva. Rio de Janeiro: Qualitymark, 1992.

GIL, A. C. Como elaborar projetos de pesquisa. 4. ed. São Paulo: Atlas, 2002.

HAIR Jr. et al. Fundamentos de métodos de pesquisa em administração. Porto Alegre: Bookman, 2005.

HOFFMAN, K. et al. Princípios de marketing de serviços: conceitos, estratégias e casos. 3. ed. São Paulo: Pioneira Thomson Learning, 2009.

INTERNATIONAL ORGANIZATION FOR STANDARDIZATION. ISO 11620:2003(F). Information et documentation. Indicateurs de performance des bibliothéques. Emenda 1: indicadores complementares de performance das bibliotecas. Genebra: ISO, 2003a.

INTERNATIONAL ORGANIZATION FOR STANDARDIZATION. ISO/TR 20983: 2003(E). Information and documentation - Performance Indicateurs for eletronic library services. Genebra: ISO, 2003b.

IGAMI, M. P. Z.; SAMPAIO, M. I. C.; VERGuEIRO, W. C. S. El uso delServqualenlaverificación de lacalidad de losservicios de unidades de información: el caso de la biblioteca del IPEN. Revista Interamericana deBibliotecologia, Medellín, Colombia, v. 28, n. 2, p. 177-191, jul./dic. 2005. Disponível em: <http://aprendeenlinea.udea.edu.co/revistas/index.php/RIB/article/view/ 8591/7936>. Acesso em: 20 out. 2013.

LANCASTER, F. W. Avaliação de serviços de bibliotecas. Brasília: Brinquet de Lemos, 1996.

LIMA, A. B. A. Estudos de usuários de bibliotecas: aproximação crítica. Revista Ciência da Informação, Brasília, v. 21, n. 3, p. 173-185, set./dez. 1992. <http://revista.ibict.br/ciinf/index.php/ciinf/article/view/1271>. Acesso em: 7 set. 2013.

LOVELOCK, C.; WRIGHT, L. Serviços: marketing e gestão. São Paulo: Saraiva, 2003.

MALHOTRA, N. K. Pesquisa de marketing: uma orientação aplicada. 4. ed. Porto Alegre: Bookman, 2006.

MORALES, M. et al. Factor structure and psychometric properties of a Spanish version of LibQUAL. Performance Measurement and Metrics, Bingley, United Kingdom, v. 12, n. 1, p. 23-37, 2011. 
PARASURAMAN, A.; ZEITHAML, V. A.; BERRY, L. L. Um modelo conceitual de qualidade de serviço e suas implicações para a pesquisa no futuro. Revista de Administração de Empresas, São Paulo, v. 46, n. 4, p. 96-108, out./dez. 2006.

PINHEIRO, L. Usuários-informação: o contexto da ciência e tecnologia. Rio de Janeiro: LTC, 1982.

REBELLO, M. A. F. R. Avaliação da qualidade dos produtos/serviços de informação: uma experiência da Biblioteca do Hospital Universitário da Universidade de São Paulo. Revista Digital de Biblioteconomia e Ciência da Informação, Campinas, v. 2, n. 1, p. 80-100, jul./dez. 2004. Disponível em: <http://www.sbu.unicamp.br/seer/ojs/index.php/rbci/article/view/304>. Acesso em: 3 set. 2012.

ROZADOS, H. B. F. Indicadores como ferramenta para avaliação de serviços de informação. In: CONGRESSO BRASILEIRO DE BIBLIOTECONOMIA, DOCUMENTAÇÃO E CIÊNCIA DA INFORMAÇÃO, 21., 2005, Curitiba. Anais... Curitiba: FEBAB, 2005. Disponível em: <http://hdl.handle.net/10183/10291>. Acesso em: 25 set. 2012.

SAMPAIO, M.I. C. et al. PAQ - Programa de avaliação da qualidadede produtos e serviços de informação: uma experiência no SIBi/USP. Ciência da Informação, Brasília, v. 33, n. 1, p. 142-148, jan./abr. 2004. Disponível em: <http://revista.ibict.br/ciinf/index.php/ciinf/article/view/70>. Acesso em: 20 out. 2013.

TAYLOR, R. S. Value-added processes in information system. Norwood, NJ: Ablex, 1986.

TRIOLA, M. F. Introdução à estatística. 10. ed., Rio de Janeiro: LTC, 2008. TRIVIÑOS, A. N. S. Introdução à pesquisa em ciências sociais: a pesquisa qualitativa em educação. São Paulo: Atlas, 1987.

VERGARA, S. C. Projetos e relatórios de pesquisa em administração. São Paulo, 2003.

VERGUEIRO, W. C. S. Qualidade em serviços de informação. São Paulo: Arte \& Ciência, 2002.

WILSON, T. D. On user studies and information needs. Journal of Documentation, v. 37, p. 3-15, 1981.

WILSON, T. D. Models in information behaviour research. Journal of Documentation, v. 55, n. 3, p. 249-270, jun. 1999.

ZEITHAML, V. A.; BITNER, M. Jo. Marketing de serviços: a empresa com foco no cliente. 2.ed. Porto Alegre: Bookman, 2003.

ZEITHAML, V. A.; BITNER, M. Jo; GREMLER, D. D. Marketing de serviços: a empresa com foco no cliente. 5. ed. Porto Alegre: Bookman, 2011. 